\title{
Frequency-modulated electromagnetic neural stimulation (FREMS) as a treatment for symptomatic diabetic neuropathy: results from a double-blind, randomised, multicentre, long-term, placebo-controlled clinical trial
}

\author{
E. Bosi $\cdot$ G. Bax $\cdot$ L. Scionti $\cdot$ V. Spallone $\cdot$ S. Tesfaye $\cdot$ \\ P. Valensi $\cdot$ D. Ziegler $\cdot$ on behalf of the FREMS \\ European Trial Study Group
}

Received: 13 August 2012 /Accepted: 6 November 2012/Published online: 13 December 2012

(C) The Author(s) 2012. This article is published with open access at Springerlink.com

\begin{abstract}
Aims/hypothesis The aim was to evaluate the efficacy and safety of transcutaneous frequency-modulated electromagnetic neural stimulation (frequency rhythmic electrical modulation system, FREMS) as a treatment for symptomatic peripheral neuropathy in patients with diabetes mellitus.

Methods This was a double-blind, randomised, multicentre, parallel-group study of three series, each of ten treatment sessions of FREMS or placebo administered within 3 weeks, 3 months apart, with an overall follow-up of about 51 weeks. The primary endpoint was the change in nerve conduction velocity (NCV) of deep peroneal, tibial and sural nerves.
\end{abstract}

Electronic supplementary material The online version of this article (doi:10.1007/s00125-012-2795-7) contains peer-reviewed but unedited supplementary material, which is available to authorised users.

FREMS Study Group members are listed in the Acknowledgements section

\section{E. Bosi $(\square)$}

Diabetes \& Endocrinology Unit, Department of Internal Medicine,

San Raffaele Hospital and San Raffaele Vita Salute University,

Via Olgettina 60,

20132 Milan, Italy

e-mail: bosi.emanuele@hsr.it

G. Bax

Servizio di Diabetologia e Dietetica,

Complesso Socio-Sanitario ai Colli, Padua, Italy

\section{Scionti}

Department of Internal Medicine, University of Perugia,

Perugia, Italy

V. Spallone

Department of Internal Medicine, University of Tor Vergata,

Rome, Italy
Secondary endpoints included the effects of treatment on pain, tactile, thermal and vibration sensations. Patients eligible to participate were aged 18-75 years with diabetes for $\geq 1$ year, $\mathrm{HbA}_{1 \mathrm{c}}<11.0 \%(97 \mathrm{mmol} / \mathrm{mol})$, with symptomatic diabetic polyneuropathy at the lower extremities (i.e. abnormal amplitude, latency or NCV of either tibial, deep peroneal or sural nerve, but with an evocable potential and measurable NCV of the sural nerve), a Michigan Diabetes Neuropathy Score $\geq 7$ and on a stable dose of medications for diabetic neuropathy in the month prior to enrolment. Data were collected in an outpatient setting. Participants were allocated to the FREMS or placebo arm (1:1 ratio)

\section{S. Tesfaye}

Diabetes Research Unit,

Sheffield Teaching Hospitals, Sheffield, UK

P. Valensi

Service d'Endocrinologie-Diabétologie-Nutrition,

Hôpital Jean Verdier, Université Paris Nord, Bondy, France

\section{Ziegler}

Institute for Clinical Diabetology, German Diabetes Center, Leibniz Center for Diabetes Research at Heinrich Heine University and Department of Metabolic Diseases, University Hospital, Düsseldorf, Germany 
according to a sequence generated by a computer random number generator, without block or stratification factors. Investigators digitised patients' date of birth and site number into an interactive voice recording system to obtain the assigned treatment. Participants, investigators conducting the trial, or people assessing the outcomes were blinded to group assignment.

Results Patients $(n=110)$ with symptomatic neuropathy were randomised to FREMS $(n=54)$ or placebo $(n=56)$. In the intention-to-treat population (50 FREMS, 51 placebo), changes in NCV of the three examined nerves were not different between FREMS and placebo (deep peroneal [means $\pm \mathrm{SE}$ ]: $0.74 \pm 0.71$ vs $0.06 \pm 1.38 \mathrm{~m} / \mathrm{s}$; tibial: $2.08 \pm$ 0.84 vs $0.61 \pm 0.43 \mathrm{~m} / \mathrm{s}$; and sural: $0.80 \pm 1.08$ vs $-0.91 \pm$ $1.13 \mathrm{~m} / \mathrm{s}$; FREMS vs placebo, respectively). FREMS induced a significant reduction in day and night pain as measured by a visual analogue scale immediately after each treatment session, although this beneficial effect was no longer measurable 3 months after treatment. Compared with the placebo group, in the FREMS group the cold sensation threshold was significantly improved, while non-significant differences were observed in the vibration and warm sensation thresholds. No relevant side effects were recorded during the study.

Conclusions/interpretation FREMS proved to be a safe treatment for symptomatic diabetic neuropathy, with immediate, although transient, reduction in pain, and no effect on NCV.

Trial registration ClinicalTrials.gov NCT01628627

Funding The clinical trial was sponsored by Lorenz Biotech (Medolla, Italy), lately Lorenz Lifetech (Ozzano dell'Emilia, Italy).

Keywords Diabetic neuropathy $\cdot$ Electrical stimulation . Nerve conduction studies $\cdot$ Neuropathic pain .

Neurostimulation therapy $\cdot$ Randomised clinical trial

$\begin{array}{ll}\text { Abbreviations } \\ \text { FREMS } & \begin{array}{l}\text { Frequency-modulated electromagnetic neural } \\ \text { stimulation (frequency rhythmic electrical } \\ \text { modulation system) }\end{array} \\ \text { ITT } & \begin{array}{l}\text { Intention to treat } \\ \text { MDNS }\end{array} \\ \text { Michigan Diabetes Neuropathy Score } \\ \text { NCV } & \text { Nerve conduction velocity } \\ \text { PP } & \text { Per protocol } \\ \text { TENS } & \text { Transcutaneous electrical nerve stimulation } \\ \text { VAS } & \text { Visual analogue scale }\end{array}$

\section{Introduction}

Peripheral diabetic neuropathy is a common and potentially disabling complication affecting a significant proportion of patients with either type 1 or type 2 diabetes [1]. The most common nerve pathological findings include endoneural capillary angiopathy and nerve ischaemia, leading to axonal atrophy and progressive loss of axons. Peripheral diabetic neuropathy affects the lower extremities, the most common clinical signs and symptoms being diminished sensation, numbness and painful symptoms, such as burning, pins and needles, intolerable pain and hyperaesthesia.

The pharmacological treatment of peripheral diabetic neuropathy remains a challenge. Different classes of agents, such as anti-epileptics (e.g. topiramate, valproic acid and the $\alpha-2-\delta$ agonists pregabalin and gabapentin), antidepressants (e.g. amitriptyline, venlafaxine, duloxetine) and opioids are variably efficacious in relieving neuropathic pain [2] but do not have an impact on the natural history of the disease. Among disease-modifying agents, the antioxidant $\alpha$-lipoic acid met secondary endpoints in a recent clinical trial in peripheral diabetic neuropathy [3] and the protein kinase $\mathrm{C}$ beta inhibitor (ruboxistaurin) was effective in a subgroup of patients with less-severe symptomatic diabetic neuropathy [4] whereas aldose reductase inhibitors were found to be ineffective [5].

Several non-pharmacological approaches, including different electrotherapies, have been proposed for the treatment of peripheral diabetic neuropathy $[6,7]$. The rationale for using electrical or magnetic stimulation is the potential enhancement of the microcirculation and endoneural blood flow, possibly counteracting the ischaemic pathogenetic component [8-10], and possibly other poorly understood mechanisms such as masking pain by interfering with pain gate control $[11,12]$.

A number of studies have reported the efficacy of various electrotherapies: transcutaneous electrical nerve stimulation (TENS); pulsed-dose electrical stimulation; peripheral nerve, nerve root, spinal cord, deep brain and epidural motor cortex stimulation; pulsed (electro-)magnetic fields and static magnetic fields; high-frequency external muscle stimulation; high-tone external muscle stimulation and external muscle stimulation [6, 7]. However, of all these electrotherapies, only TENS is currently recommended as a treatment for painful peripheral diabetic neuropathy by the American Academy of Neurology $[2,13]$.

Recently, a novel transcutaneous frequency-modulated electromagnetic neural stimulation (also known as frequency rhythmic electrical modulation system, FREMS), has been developed, using an innovative mechanism of stimulation, consisting of a sequence of modulated electrical stimuli that varies automatically in terms of pulse frequency, duration and voltage amplitude. In a pilot, randomised, crossover study, FREMS reduced diabetic neuropathy pain and ameliorated the sensory tactile and vibration perception threshold and motor nerve conduction velocity (NCV) when compared with placebo [14]. 
The aim of this study was to test the efficacy and safety of FREMS in a randomised, double-blind, placebo-controlled multicentre study enrolling a large population of patients with symptomatic diabetic polyneuropathy, with repeated treatment sessions and a post-treatment follow-up of adequate length.

\section{Methods}

Participants Patients meeting the following criteria were invited to participate in this study: (1) documented diagnosis of type 1 or type 2 diabetes according to ADA criteria [15], with duration of disease of at least 1 year and $\mathrm{HbA}_{1 \mathrm{c}}<11.0 \%$ (97 mmol $/ \mathrm{mol}$ ) (normal range 3.5-6.0\%, 15-42 mmol/mol); (2) age between 18 and 75 years; (3) symptomatic diabetic polyneuropathy affecting the lower extremities with at least one positive sensory symptom such as pain, burning, paraesthesia or prickling; (4) abnormalities in amplitude $(<6 \mathrm{mV})$, latency $(>6.5 \mathrm{~ms})$ or conduction velocity $(<40 \mathrm{~m} / \mathrm{s})$ in at least one motor nerve (either tibial or deep peroneal) and/or in the sural nerve; (5) a measurable sensitive NCV and evocable potential in the sural nerve; (6) a Michigan Diabetes Neuropathy Score (MDNS) [16] equal to or greater than 7 points; and (7) stable dose of pain medications or other medications prescribed for diabetic neuropathy, if any, during the month leading up to enrolment. Exclusion criteria were: (1) previous treatment with TENS or other electrotherapy for diabetic neuropathy; (2) presence of an implanted pacemaker, defibrillator or neurostimulator; (3) presence of an active foot ulcer and/or previous major amputation of the lower extremities; and (4) any concomitant severe disease limiting compliance to study procedures or life expectancy.

Study design The study was designed as a randomised, double-blind, placebo-controlled multicentre trial. After screening, eligible participants were randomised to the FREMS or placebo arm with a 1:1 ratio according to a sequence generated by a computer random number generator, without block or stratification factors. Investigators digitised patients' date of birth and site number into an interactive voice recording system to obtain the assigned treatment. Treatment group was confirmed by a fax, which was included in the case-report form. Randomised patients received three series of FREMS or placebo treatment at 3-month (12-13 weeks) intervals: each series consisted of ten consecutive treatment sessions administered at least $24 \mathrm{~h}$ apart, within a maximum of 21 days. Follow-up after randomisation was 51 weeks. For the entire duration of the study each patient was examined by the same investigator who was also responsible for the administration of the study questionnaires and reporting of side effects. Patients, clinical investigators, technicians, neurophysiologists and statisticians were unaware of treatment assignment. Moreover, to secure the blindness of the study, patients were instructed not to consider any perception at the site of electrode placement during the treatment as a sign of active treatment and also not to judge the absence of any such perception as a sign of sham treatment. Investigators who performed the neurological outcome assessment were different from those who administered the FREMS or placebo treatment. Patients were enrolled at six clinical sites: Milan, Padua, Perugia and Rome (Italy), Düsseldorf (Germany) and Bondy (France). The study protocol was approved by the Ethics Committee of each participating Institution and written informed consent was obtained from all participants before enrolment.

Intervention Treatment with FREMS consisted of sequences of biphasic (negative and positive), asymmetric and electrically balanced pulses composed of an active phase of high negative voltage spike (variable, $\max -300 \mathrm{~V}$ ) and extra short duration (variable, $10-100 \mu \mathrm{s}$, mostly $\sim 40 \mu \mathrm{s}$ ) followed by a recharging phase of low voltage and long duration (0.9-999 ms); the pulse frequency was variable, in the range of $1-1,000 \mathrm{~Hz}$ (mainly in the low range [1-50 Hz]; see electronic supplementary material [ESM] Fig. 1). Treatment with placebo consisted of no electrical pulses (i.e., sham treatment). Both FREMS and placebo treatment were administered using the Aptiva device (Lorenz Lifetech, Ozzano dell'Emilia [formerly Lorenz Biotech, Medolla], Italy) via four pairs of disposable electrodes applied to both lower extremities. For the purpose of this study the original device was modified by adding a switch to apply treatment A (later revealed to be placebo) or treatment $\mathrm{B}$ (later revealed to be FREMS). Each session of either placebo or FREMS treatment lasted $30 \mathrm{~min}$ and during sessions patients were invited to modulate the delivery of sham or true neurostimulation by progressively increasing the voltage of the electrical stimulation from $0 \mathrm{~V}$ to $255 \mathrm{~V}$ through a hand-held remote control, which increased the nominal voltage by $1 \mathrm{~V}$ at each step. The complete treatment consisted of three series of ten consecutive (one a day for 5 days/week) FREMS or placebo treatments to both lower limbs.

Timing of study assessments Participants were assessed eight times during the study: $\mathrm{T}-1$ (enrolment visit), $\mathrm{T} 0$ (week 0 , within 2 weeks after $\mathrm{T}-1$ : start of first therapeutic cycle), T1 (week 3, after the first therapeutic cycle), T2 (week 17: start of second therapeutic cycle), T3 (week 20, after the second therapeutic cycle), T4 (week 34: start of third therapeutic cycle), T5 (week 37, after the third therapeutic cycle) and T6 (week 51).

Neurophysiology Neurophysiological measurements were carried out at $\mathrm{T}-1, \mathrm{~T} 1, \mathrm{~T} 2, \mathrm{~T} 4$ and $\mathrm{T} 6$. Before the start of 
the study a common operative protocol for the electroneurophysiological measurements was developed by a panel chaired by the Neurophysiology Coordinating Centre, responsible for the central reading of all neurophysiological measurements, and formed by all the neurophysiologists responsible for the neurophysiological measurements at study centres. The panel was based at the San Raffaele Hospital in Milan, but panel members were not involved in the conduction of the clinical trial. The protocol detailed the devices to be used, the test procedures and the environmental conditions required to obtain standardised study measurements. Neurophysiological variables recorded locally were transmitted to the Neurophysiology Coordinating Centre for reading, validation and data quality assurance. Measurements included motor NCV of the tibial and deep peroneal nerves, and sensory NCV of the sural nerve. The $\mathrm{z}$ scores of conduction velocity, latency, amplitude and $\mathrm{F}$ wave were also measured in all three nerves.

Pain Pain was assessed at T0, T1, T2, T3, T4, T5 and T6. Patients were asked to assess daytime and night-time pain intensity during the $24 \mathrm{~h}$ preceding each outpatient clinic visit using a $0-100 \mathrm{~mm}$ visual analogue scale (VAS). Results were expressed as absolute values [17, 18] and as the percentage of responders [19]; patients were defined as responders if they had $\mathrm{a} \geq 30 \%$ or $\geq 50 \%$ reduction in nighttime or daytime pain score at the end of each treatment cycle compared with their pre-cycle score (i.e., T1 vs T0, T3 vs T2, T5 vs T4).

Tactile sensation Tactile sensation was assessed at $\mathrm{T} 0, \mathrm{~T} 1$, T2, T4 and T6 using the $5.07(10 \mathrm{~g})$ Semmes-Weinstein monofilament test. We used a six-sensitive-point test protocol, with assessment at the big toe, third and fifth toe and three (first, third and fifth) metatarsal head points on both feet, with three examinations per point, including one random false stimulation. Results were expressed as an index calculated by dividing the number 6 (i.e., the total number of potentially sensitive points) by the number of points that tested positive [20, 21].

Vibration perception threshold Foot vibration perception threshold was assessed at T0, T1, T2, T4 and T6 using a commercially available vibratory sensory analyser device (MEDOC VSA 3000; Medoc Advanced Medical Systems, Durham, NC, USA) [22]. Results were expressed in micrometres.

Thermal sensation thresholds At T0, T1, T2, T4 and T6, cold and warm temperature sensation thresholds were measured using a commercially available thermotesting device (MEDOC TSA-II, Medoc Advanced Medical Systems) [23]. Results were expressed in degree centigrade.
Neurological clinical examination The MDNS was used for grading the severity of neuropathic deficits [16] at T-1, T1, $\mathrm{T} 2, \mathrm{~T} 4$ and $\mathrm{T} 6$.

Laboratory testing Routine haematology and biochemistry, including fasting plasma glucose and $\mathrm{HbA}_{1 \mathrm{c}}$, were measured locally at each participating centre (at T0, T1, T2, T4 and T6). Local $\mathrm{HbA}_{1 \mathrm{c}}$ assays were aligned to the assay used in the DCCT.

Study endpoints The primary endpoint of the study was the change in NCV in either the tibial, deep peroneal or sural nerve at the end of three series of FREMS or placebo treatment (T5), compared with baseline. Secondary endpoints were the changes at $\mathrm{T} 5$ vs baseline in: tactile sensation by $10 \mathrm{~g}$ monofilament; vibration perception, cold and warm temperature sensation thresholds; MDNS and changes in daytime and night-time pain intensity assessed by VAS after each treatment series.

Statistical analysis Statistical analysis was conducted using Stata 9.1 software (StataCorp, College Station, TX, USA). Continuous variables with a normal distribution were compared in the two treatment groups using the two-sided Student's $t$ test; variables with a non-normal distribution were compared using the Mann-Whitney test. Categorical variables were compared using the $\chi^{2}$ test or Fisher's exact test when appropriate. General mixed-effects models were used to analyse longitudinal data and compare the effect of treatment between the two groups. Treatment group, centre, visit and the treatment group-by-visit interaction were considered as fixed effects and baseline, sex and height as covariates. A two-sided test with a $p$ value $<0.05$ was considered statistically significant. Bonferroni correction was used to account for multiple comparisons. Primary and secondary endpoints were analysed for a 'practical' intent-to-treat (ITT) population [24], which consisted of all randomised patients with a baseline and at least one postbaseline assessment. Primary and secondary endpoints were also analysed in the per-protocol (PP) population, which consisted of all randomised patients included in the ITT population who completed the study without major protocol violations.

\section{Results}

General characteristics of study participants Of the 164 screened patients, 54 individuals were found ineligible mainly because NCV in the sural nerve could not be measured. The remaining 110 eligible patients were randomly assigned to receive placebo $(n=56)$ or FREMS $(n=54)$ (Fig. 1). One-hundred-and-one participants $(n=51$ in the 
Fig. 1 Flow diagram of the study

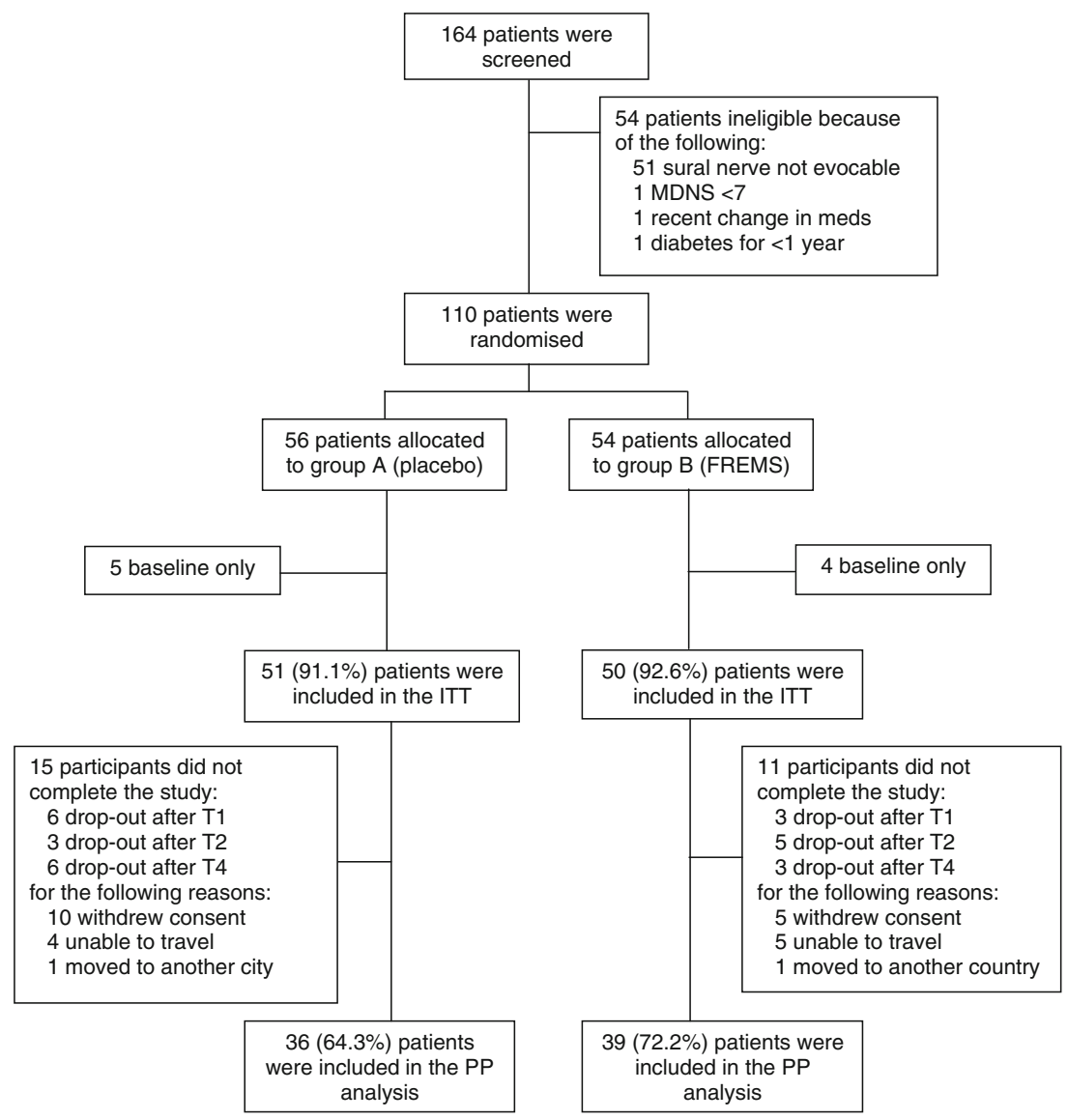

placebo group and $n=50$ in the FREMS group) had at least one follow-up visit after randomisation and were included in the ITT population (practical ITT). Seventy-five participants completed the study through to T6 (39 in the placebo group and 36 in the FREMS group) and were also included in the PP population. The most common reason for not completing the study was the burden of repeated hospital visits for the FREMS/placebo treatment sessions in individuals with a certain degree of disability. No participant dropped out from the study because of treatment-related side effects. General characteristics of study participants at baseline by the assigned treatment group are reported in Table 1. Baseline variables were not significantly different between the two treatment groups.

NCV (primary endpoint) No centre heterogeneity in the response to treatment was detected. The primary outcome of the study was not met since in the ITT population the change in NCV at T6 vs baseline was not significantly different between treatment groups for any of the three examined nerves (Table 2). Similar results were observed in the PP population, with the exception of the sural nerve where the NCV increase was marginally significant ( $p=$ 0.049 FREMS vs placebo) (Table 2, ESM Fig. 2). Furthermore, no differences in NCV z score, latency, amplitude and
F wave were observed during the study between the FREMS and placebo group, for any of the three examined nerves.

Pain Of the 110 randomised study participants, 88 reported pain, during either the day or the night, as part of their symptomatic neuropathy; all randomised patients were considered in the analysis of pain, including those who had VAS score 0 at baseline (18 had VAS score 0 at night, 13 had VAS score 0 in the day, with nine patients having VAS score 0 for both night and day). In the ITT population both night-time pain (Fig. 2a) and daytime pain (Fig. 2b) measured as VAS were significantly reduced in the FREMS group compared with the placebo group at T1 (both $p<$ $0.001)$, T3 (both $p<0.001)$ and T5 $(p<0.001$ and $p=0.02$, respectively) (i.e. at the time of completion of each of the three treatment series). The beneficial effect of FREMS on both daytime and night-time pain was not detectable 3 months after the end of the last treatment series. Similar results were observed in the PP population (data not shown). The rate of responders with either $\geq 30 \%$ or $\geq 50 \%$ reduction in night-time or daytime pain score was significantly higher in patients treated with FREMS compared with placebo after the second and the third treatment cycle, while nonsignificant differences were observed after the first cycle (ESM Table 1). 
Table 1 Baseline characteristics of the study participants (ITT population)

\begin{tabular}{|c|c|c|}
\hline Baseline characteristic & Placebo $(n=51)$ & FREMS $(n=50)$ \\
\hline Sex, \% women & $39(25.8,53.9)$ & $28(16.2,42.3)$ \\
\hline Age, years & $61.3 \pm 8.3$ & $59.0 \pm 10.6$ \\
\hline \multicolumn{3}{|l|}{ Diabetes, No. (\%) } \\
\hline Type 1 & $9(17.6)$ & $10(20)$ \\
\hline Type 2 & $42(82.4)$ & $40(80)$ \\
\hline Duration of diabetes, years & $12(8,25)$ & $13(10,18)$ \\
\hline BMI, $\mathrm{kg} / \mathrm{m}^{2}$ & $28.5 \pm 4.8$ & $28.8 \pm 4.8$ \\
\hline \multicolumn{3}{|l|}{$\mathrm{HbA}_{1 \mathrm{c}}{ }^{\mathrm{a}}$} \\
\hline$\%$ & $7.6 \pm 1.2$ & $7.9 \pm 1.2$ \\
\hline $\mathrm{mmol} / \mathrm{mol}$ & $59.8 \pm 13.0$ & $63.1 \pm 13.4$ \\
\hline \multicolumn{3}{|l|}{ Diabetes treatment, $\%$} \\
\hline Lifestyle modification & $18(8.4,30.9)$ & $14(5.8,26.7)$ \\
\hline Oral agents & $29(17.5,43.8)$ & $44(30.0,58.7)$ \\
\hline Oral agents and insulin & $18(8.4,30.9)$ & $8(2.2,19.2)$ \\
\hline Insulin & $35(22.4,50.0)$ & $34(21.2,48.8)$ \\
\hline Serum creatinine, $\mu \mathrm{mol} / 1$ & $83.1 \pm 18.5$ & $85.7 \pm 24.7$ \\
\hline MDNS & $13.7 \pm 5.0$ & $11.6 \pm 4.6$ \\
\hline Night-time pain, VAS score & $45.2 \pm 29.6$ & $41.3 \pm 29.7$ \\
\hline Daytime pain, VAS score & $40.9 \pm 24.0$ & $31.6 \pm 26.3$ \\
\hline $\begin{array}{l}\text { Vibration perception } \\
\text { threshold, } \mu \mathrm{m}\end{array}$ & $22.4 \pm 23.1$ & $24.2 \pm 26.5$ \\
\hline Warm sensation threshold, ${ }^{\circ} \mathrm{C}$ & $38.0 \pm 9.3$ & $38.5 \pm 9.2$ \\
\hline Cold sensation threshold, ${ }^{\circ} \mathrm{C}$ & $26.5 \pm 7.6$ & $26.0 \pm 7.7$ \\
\hline \multicolumn{3}{|l|}{$\mathrm{NCV}, \mathrm{m} / \mathrm{s}$} \\
\hline Sural nerve & $42.2 \pm 6.3$ & $42.0 \pm 4.3$ \\
\hline Tibial nerve & $42.3 \pm 4.6$ & $40.1 \pm 6.8$ \\
\hline Deep peroneal nerve & $43.8 \pm 4.8$ & $41.5 \pm 5.0$ \\
\hline \multicolumn{3}{|l|}{ Amplitude } \\
\hline Sural nerve, $\mu \mathrm{V}$ & $4.98 \pm 3.29$ & $4.82 \pm 3.91$ \\
\hline Tibial nerve, $\mathrm{mV}$ & $10.47 \pm 5.82$ & $8.59 \pm 5.87$ \\
\hline Deep peroneal nerve, $\mathrm{mV}$ & $5.43 \pm 3.28$ & $4.80 \pm 3.37$ \\
\hline
\end{tabular}

Continuous variables are presented as mean $\pm \mathrm{SD}$ and categorical variables as percentage $(95 \% \mathrm{CI})$

${ }^{\mathrm{a}}$ The normal range for $\mathrm{HbA}_{1 \mathrm{c}}$ is $3.5-6.0 \%(15-42 \mathrm{mmol} / \mathrm{mol})$.

At baseline 14 (26\%) participants in the FREMS group and $9(16 \%)$ in the placebo group were taking medication(s) for painful neuropathy (NS). During follow-up, four additional participants ( $n=3$ in the placebo group, $n=1$ in the FREMS group) were prescribed medication for painful neuropathy, while none of the participants' medications were discontinued.

Tactile sensation There were no significant differences in the changes of tactile sensation between FREMS and placebo groups.

Vibration and thermal sensation thresholds A significant increase in the cold sensation threshold was observed in
Table 2 Adjusted mean change in NCV at T6 vs baseline for the deep peroneal, tibial and sural nerve in the ITT and PP populations

\begin{tabular}{llcl}
\hline Examined nerve & \multicolumn{2}{l}{$\begin{array}{l}\text { Adjusted mean change in NCV }(\mathrm{m} / \mathrm{s}) \\
\text { at T6 vs baseline }\end{array}$} & \\
\cline { 2 - 3 } & FREMS & Palue \\
& & & \\
\hline ITT population & & $0.06 \pm 1.38$ & $\mathrm{NS}$ \\
Deep peroneal & $0.74 \pm 0.71$ & $0.61 \pm 0.43$ & $\mathrm{NS}$ \\
Tibial & $2.08 \pm 0.84$ & $-0.91 \pm 1.13$ & $\mathrm{NS}$ \\
Sural & $0.80 \pm 1.08$ & & \\
PP population & & $-0.05 \pm 0.44$ & 0.049 \\
Deep peroneal & $0.98 \pm 0.72$ & $0.58 \pm 0.46$ & $\mathrm{NS}$ \\
Tibial & $0.76 \pm 0.59$ & $0.44 \pm 0.96$ & $\mathrm{NS}$ \\
Sural & $1.13 \pm 0.87$ & & \\
\hline
\end{tabular}

Data are presented as means \pm SE. ITT population: $n=50$ (FREMS) and $n=51$ (placebo); PP population: $n=39$ (FREMS) and $n=36$ (placebo).

${ }^{\mathrm{a}}$ General mixed-effects model analysis

the FREMS group compared with the placebo group, both in the ITT (adjusted mean change at T5 vs baseline $2.3 \pm 1.4$ vs $-1.0 \pm 1.2^{\circ} \mathrm{C}$, FREMS vs placebo; $\left.p=0.041\right)$ and $\mathrm{PP}(0.8 \pm$ 0.9 vs $-0.05 \pm 0.9 \mathrm{C}$, FREMS vs placebo; $p=0.008$ )
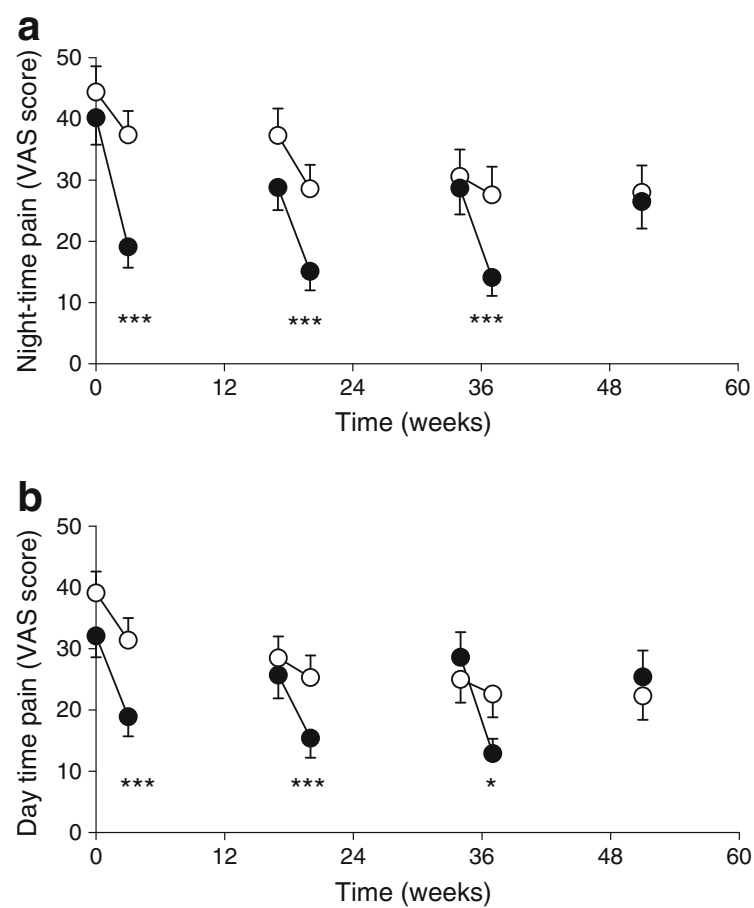

Fig. 2 VAS score for night-time pain (a) and daytime pain (b) for study participants in the FREMS group (black circles) and in the placebo group (white circles) in the ITT population, before and after each treatment cycle and at the end of follow-up. Data are presented as means and SE. For each treatment cycle we compared the difference in the VAS score (end of treatment cycle - beginning of treatment cycle) between treatment groups: ${ }^{*} p<0.05,{ }^{* *} p<0.001$, FREMS vs placebo 
populations. Only non-significant trends were observed in the FREMS group compared with the placebo group for the warm sensation and the vibration threshold.

MDNS There were no significant differences in the changes in MDNS between the two treatment groups.

Diabetes control and kidney function During the study $\mathrm{HbA}_{1 \mathrm{c}}$ and serum creatinine did not change in either treatment group.

Safety No treatment-related severe or non-severe adverse events were recorded during the study, either in the FREMS or in the placebo group. Patients reported only a very slight burning sensation at the site of electrode placement during the series of treatments later revealed as FREMS, with no residual skin signs. No particular perception was recorded during placebo sessions.

\section{Discussion}

This study investigated the safety and efficacy of FREMS in a randomised, double-blind, placebo-controlled, parallelgroup, multicentre clinical trial, one of the largest and longest clinical trials ever performed to test an electrotherapy in peripheral diabetic neuropathy. FREMS had no significant effect on the NCV of the peroneal, tibial or sural nerves (the primary endpoint of the study), was safe and resulted in a significant reduction in both daytime and night-time pain in patients with symptomatic diabetic polyneuropathy.

The effect of FREMS on the pain of diabetic polyneuropathy was notable, with significantly reduced VAS score at the end of all three FREMS treatment series for both nighttime and daytime pain, compared with placebo. However, the beneficial effect on pain was not sustained, since the VAS score for both night-time pain and daytime pain had returned toward the baseline value 3 months after the end of the last FREMS treatment series, possibly suggesting the need for repeated treatments to maintain a clinically meaningful effect on pain over time. On the other hand, it should be emphasised that, compared with pharmacological treatment for neuropathic pain, FREMS shows a proportion of $50 \%$ responders, as high as the first-line drugs duloxetine and pregabalin [25], with the advantage of having no relevant side effects, and requiring intermittent instead of daily treatment. In fact, although patients reported some perceptions at the site of electrode placement during treatment later revealed as FREMS, and this sensation was not perceived by patients randomised to receive placebo treatment, no real side effects were associated with FREMS. Moreover, although the dropout rate during the study was not negligible, it was not treatment related, as suggested by the fact that it was higher in the placebo arm than in the FREMS arm (35.7\% and $27.8 \%$, respectively) and caused by the burden associated with study participation.

This clinical trial confirms the efficacy of FREMS in reducing the pain of diabetic polyneuropathy, as previously reported, but replicates only in part the findings of the pilot study in which NCV, tactile sensation and vibration perception thresholds were also significantly improved [14]. In particular NCV, the primary endpoint of this study, is unchanged after FREMS treatment. These discrepancies are likely explained, at least in part, by the different severity of diabetic polyneuropathy in the two study populations. The participants in the early pilot study had severe peripheral diabetic neuropathy, with significantly reduced NCVs (motor $\mathrm{NCV}$ was $35-36 \mathrm{~m} / \mathrm{s}$; sensory NCV was $27-29 \mathrm{~m} / \mathrm{s}$ ) and many patients were without evocable potentials (motor NCV was measurable in $84 \%$, sensory NCV in $48 \%$ of participants) [14]. For participants to be eligible for inclusion in the present trial, NCV had to be measurable for all the three nerves investigated (deep peroneal, tibial and sural). This led to the selection of individuals with a less-severe diabetic neuropathy with only mild impairment of NCVs at baseline (mean NCVs in the range of 40.1-43.8 m/s; Table 1) which, in turn, leaves a limited margin for improvement in response to an active treatment, thus requiring a higher number of patients to show significant differences. The relatively low proportion of patients taking specific medications for painful neuropathy at baseline, with few prescription changes during follow-up, also reflects a less-severe diabetic neuropathy in the patients enrolled in this study. Therefore, patients with severe neuropathy are likely to benefit from FREMS more than patients with milder impairment of NCV.

The effects of FREMS on thermal, vibration and tactile sensations were also inconclusive, with a significant improvement of cold sensation thresholds and non-significant effects on other variables.

FREMS, being based on TENS methodology, falls within the generic definition of TENS; nevertheless, the electrical stimulus in FREMS significantly differs from those commonly used in other known electrotherapies, mainly TENS apparatuses. FREMS provides sequences of biphasic electrical stimuli that vary simultaneously and automatically in frequency, pulse duration and amplitude, reaching relatively high intensity $(300 \mathrm{~V})$ in association with a very short duration $(10-100 \mu \mathrm{s})$, maintaining electrical balance in the tissues [26]. This feature is novel with respect to existing electrotherapies, which are normally based on 'geometrical' waveforms characterised by lower peak intensity and higher pulse duration. Regarding FREMS, it should be emphasised that specific new mechanisms of action have been described, such as enhancement of microvascular blood flow measured by laser doppler flowmetry [27], increase in vasomotor activity mediated by smooth cells [28] and release 
of vascular endothelial growth factor [29]. These reports collectively suggest a possible improvement in endoneural blood flow induced by FREMS to explain its effects on neuropathy. Due to lack of data, it is not known at present whether and to what extent the basic mechanism of FREMS is similar to or different from those underlying TENS and other electrotherapies; in fact, although the latter have long been investigated and used in clinical practice, the foundation of their therapeutic effect is unknown and largely assumed to be based on interference with the pain gate control [12]. Future studies will hopefully clarify this important issue.

A relevant aspect to take into account when considering any new treatment is cost. In this context the cost of FREMS is similar to that of TENS and likely lower than that of the most expensive pharmacological treatments. However, proper cost-effectiveness studies of different treatments for diabetic neuropathy are still lacking and should be conducted in the future, as recently recommended [2].

A strength of this study is its design, which included double-blind masking, a placebo arm, three repeated series of treatment and an overall follow-up period of almost 1 year. These design features are quite unique in the spectrum of the clinical trials testing electrotherapies for diabetic neuropathy. A limitation of our study is that the criteria for inclusion did select patients with relatively mild symptomatic painful neuropathy and, therefore, our finding may not apply to patients with more severe diabetic neuropathy.

In conclusion, the findings of this study confirm that FREMS is safe and leads to pain relief and improvement in cold sensation threshold in diabetic patients with symptomatic polyneuropathy.

Acknowledgements Members of the FREMS European Trial Study Group panel for the measurement of electrophysiological variables were G. Comi, U. del Carro, P. Morana (Neurophysiology Coordinating Centre, San Raffaele Hospital \& Scientific Institute, Milan, Italy), M. Ferullo, L. Zanetti, S. Bassan (Servizio di Diabetologia e Dietetica, Complesso Socio-Sanitario ai Colli, Padua, Italy), A. Bartocci, E. Venturini (Department of Internal Medicine, University of Perugia, Italy), G. A. Marfia, C. Pachaz (Department of Internal Medicine, University of Tor Vergata, Rome, Italy), A.-L. Frenkel (Service d'Endocrinologie-Diabétologie-Nutrition, Hôpital Jean Verdier, Université Paris Nord, Bondy, France), M. Behler and M. Schroers-Teuber (Institute for Clinical Diabetology, German Diabetes Center, Leibniz Center for Diabetes Research at Heinrich Heine University and Department of Metabolic Diseases, University Hospital, Düsseldorf, Germany). Local investigators for the FREMS European Trial Study Group included E. Bosi, G. Galimberti, E. Peretti (Diabetes \& Endocrinology Unit, Department of Internal Medicine, San Raffaele Hospital and San Raffaele Vita Salute University, Milan, Italy), G. Bax (Servizio di Diabetologia e Dietetica, Complesso Socio-Sanitario ai Colli, Padua, Italy), L. Scionti, F. Notarstefano, A. Mastroianni, F. Scarlato (Department of Internal Medicine, University of Perugia, Italy), V. Spallone, R. Morganti, C. D'Amato, L. Cacciotti (Department of Internal Medicine, University of Tor Vergata, Rome, Italy), P. Valensi, S. Chiheb (Service d'Endocrinologie-Diabétologie-Nutrition, Hôpital Jean Verdier, Université Paris Nord, Bondy, France) and D. Ziegler (Institute for Clinical Diabetology, German Diabetes Center, Leibniz Center for Diabetes
Research at Heinrich Heine University and Department of Metabolic Diseases, University Hospital, Düsseldorf, Germany).

We thank F. de Terlizzi (independent biostatistician, Italy) for statistical analysis and M. Scavini (Diabetes Research Institute, San Raffaele Hospital \& Scientific Institute, Milan, Italy) for help in statistical analysis and critical review of the manuscript.

Duality of interest E. Bosi and G. Bax received lecture fees from Lorenz Biotech, lately Lorenz Lifetech, sponsor of the study and manufacturer of the device delivering FREMS electrotherapy.

Contribution statement EB contributed to the study design and conduction, data analysis, and wrote the manuscript. GB contributed to the study conduction and data analysis and reviewed the manuscript. LS, VS, PV and DZ contributed to the study design and conduction and reviewed the manuscript. ST contributed to the study design and reviewed the manuscript. All authors approved the final version of the manuscript.

Open Access This article is distributed under the terms of the Creative Commons Attribution Noncommercial License which permits any noncommercial use, distribution, and reproduction in any medium, provided the original author(s) and the source are credited.

\section{References}

1. Tesfaye S, Boulton AJ, Dyck PJ et al (2010) Diabetic neuropathies: update on definitions, diagnostic criteria, estimation of severity, and treatments. Diabetes Care 33:2285-2293

2. Bril V, England J, Franklin GM et al (2011) Evidence-based guideline: treatment of painful diabetic neuropathy: report of the American Academy of Neurology, the American Association of Neuromuscular and Electrodiagnostic Medicine, and the American Academy of Physical Medicine and Rehabilitation. Neurology 76:1758-1765

3. Ziegler D, Low PA, Litchy WJ et al (2011) Efficacy and safety of antioxidant treatment with $\alpha$-lipoic acid over 4 years in diabetic polyneuropathy: the NATHAN 1 trial. Diabetes Care 34:2054 2060

4. Vinik AI, Bril V, Kempler P et al (2005) Treatment of symptomatic diabetic peripheral neuropathy with the protein kinase $\mathrm{C}$ betainhibitor ruboxistaurin mesylate during a 1-year, randomized, placebo-controlled, double-blind clinical trial. Clin Ther 27:1164-1180

5. Chalk C, Benstead TJ, Moore F (2007) Aldose reductase inhibitors for the treatment of diabetic polyneuropathy. Cochrane Database Syst Rev: Art, no.: CD004572. doi:10.1002/14651858.CD004572.pub2

6. Cruccu G, Aziz TZ, Garcia-Larrea L et al (2007) EFNS guidelines on neurostimulation therapy for neuropathic pain. Eur J Neurol 14:952-970

7. Pieber K, Herceg M, Paternostro-Sluga T (2010) Electrotherapy for the treatment of painful diabetic peripheral neuropathy: a review. J Rehabil Med 42:289-295

8. Gilcreast DM, Stotts NA, Froelicher ES, Baker LL, Moss KM (1998) Effect of electrical stimulation on foot skin perfusion in persons with or at risk for diabetic foot ulcers. Wound Repair Regen 6:434-441

9. Kaada B (1982) Vasodilation induced by transcutaneous nerve stimulation in peripheral ischemia: Raynaud's phenomenon and diabetic polyneuropathy. Eur Heart J 3:303-314

10. Jacobs MJ, Jorning PJ, Joshi SR, Kitslaar PJ, Slaaf DW, Reneman RS (1988) Epidural spinal cord electrical stimulation improves microvascular blood flow in severe limb ischemia. Ann Surg 207:179-183 
11. Armstrong DG, Lavery LA, Fleischli JG, Gilham KA (1997) Is electrical stimulation effective in reducing neuropathic pain in patients with diabetes? J Foot Ankle Surg 36:260-263

12. Melzack R, Wall PD (1965) Pain mechanisms: a new theory. Science 150:971-979

13. Dubinsky RM, Miyasaki J (2010) Assessment: efficacy of transcutaneous electric nerve stimulation in the treatment of pain in neurologic disorders (an evidence-based review). Report of the Therapeutics and Technology Assessment Subcommittee of the American Academy of Neurology. Neurology 74:173-176

14. Bosi E, Conti M, Vermigli C et al (2005) Effectiveness of frequency-modulated electromagnetic neural stimulation in the treatment of painful diabetic neuropathy. Diabetologia 48:817-823

15. American Diabetes Association (2012) Diagnosis and classification of diabetes mellitus. Diabetes Care 35(Suppl 1):S64-S71

16. Feldman EL, Stevens MJ, Thomas PK, Brown MB, Canal N, Greene DA (1994) A practical two-step quantitative clinical and electrophysiological assessment for the diagnosis and staging of diabetic neuropathy. Diabetes Care 17:1281-1289

17. Ohnhaus EE, Adler R (1975) Methodological problems in the measurement of pain: a comparison between the verbal rating scale and the visual analogue scale. Pain 1:379-384

18. Cruccu G, Anand P, Attal N et al (2004) EFNS guidelines on neuropathic pain assessment. Eur J Neurol 11:153-162

19. Dworkin RH, Turk DC, Wyrwich KW et al (2008) Interpreting the clinical importance of treatment outcomes in chronic pain clinical trials: IMMPACT recommendations. J Pain 9:105-121

20. Kumar S, Fernando DJ, Veves A, Knowles EA, Young MJ, Boulton AJ (1991) Semmes-Weinstein monofilaments: a simple, effective and inexpensive screening device for identifying diabetic patients at risk of foot ulceration. Diabetes Res Clin Pract 13:63-67
21. Perkins BA, Bril V (2003) Diabetic neuropathy: a review emphasizing diagnostic methods. Clin Neurophysiol 114:1167-1175

22. Strotmeyer ES, de Rekeneire N, Schwartz AV et al (2008) The relationship of reduced peripheral nerve function and diabetes with physical performance in older white and black adults. The Health, Aging, and Body Composition (Health ABC) Study. Diabetes Care 31:1767-1772

23. Zinman LH, Bril V, Perkins BA (2004) Cooling detection thresholds in the assessment of diabetic sensory polyneuropathy: comparison of CASE IV and Medoc instruments. Diabetes Care 27:1674-1679

24. Gillings DB, Koch GG (1991) The application of the principle of intention-to-treat to the analysis of clinical trials. Drug Inform J $25: 411-424$

25. Spallone V, Lacerenza M, Rossi A, Sicuteri R, Marchettini P (2012) Painful diabetic polyneuropathy: approach to diagnosis and management. Clin J Pain 28:726-743

26. Barrella M, Toscano R, Goldoni M, Bevilacqua M (2007) Frequency rhythmic electrical modulation system (FREMS) on $\mathrm{H}-$ reflex amplitudes in healthy subjects. Eura Medicophys 43:37-47

27. Conti M, Peretti E, Cazzetta G et al (2009) Frequency-modulated electromagnetic neural stimulation enhances cutaneous microvascular flow in patients with diabetic neuropathy. J Diabetes Complications 23:46-48

28. Bocchi L, Evangelisti A, Barrella M, Scatizzi L, Bevilacqua M (2010) Recovery of $0.1 \mathrm{~Hz}$ microvascular skin blood flow in dysautonomic diabetic (type 2) neuropathy by using Frequency Rhythmic Electrical Modulation System (FREMS). Med Eng Phys 32:407-413

29. Bevilacqua M, Dominguez LJ, Barrella M, Barbagallo M (2007) Induction of vascular endothelial growth factor release by transcutaneous frequency modulated neural stimulation in diabetic polyneuropathy. J Endocrinol Invest 30:944-947 